\title{
Sensorimotor Reorganizations of Arm Kinematics and Postural Strategy for Functional Whole-Body Reaching Movements in Microgravity
}

\author{
Thomas Macaluso ${ }^{1}$, Christophe Bourdin ${ }^{1}$, Frank Buloup ${ }^{1}$, Marie-Laure Mille 1, 2, 3 , \\ Patrick Sainton ${ }^{1}$, Fabrice R. Sarlegna ${ }^{1}$, Jean-Louis Vercher ${ }^{1}$ and Lionel Bringoux ${ }^{1 *}$ \\ ${ }^{1}$ Aix Marseille Univ, CNRS, ISM, Marseille, France, ${ }^{2}$ UFR STAPS, Université de Toulon, La Garde, France, ${ }^{3}$ Department of \\ Physical Therapy and Human Movement Sciences, Feinberg School of Medicine, Northwestern University, Chicago, IL, \\ United States
}

OPEN ACCESS

Edited by:

Olivier White,

INSERM U1093, Université de Bourgogne Franche Comté, France

Reviewed by:

Francesco Lacquaniti, Università degli Studi di Roma Tor Vergata, Italy

Vaughan G. Macefield, Mohammed Bin Rashid University of Medicine and Health Sciences, United Arab Emirates

*Correspondence: Lionel Bringoux lionel.bringoux@univ-amu.fr

Specialty section: This article was submitted to Integrative Physiology a section of the journal Frontiers in Physiology

Received: 27 June 2017 Accepted: 05 October 2017 Published: 20 October 2017

Citation: Macaluso T, Bourdin C, Buloup F, Mille M-L, Sainton P, Sarlegna FR, Vercher J-L and Bringoux L (2017) Sensorimotor Reorganizations of Arm Kinematics and Postural Strategy for

Functional Whole-Body Reaching Movements in Microgravity. Front. Physiol. 8:821 doi: 10.3389/fphys.2017.00821
Understanding the impact of weightlessness on human behavior during the forthcoming long-term space missions is of critical importance, especially when considering the efficiency of goal-directed movements in these unusual environments. Several studies provided a large set of evidence that gravity is taken into account during the planning stage of arm reaching movements to optimally anticipate its consequence upon the moving limbs. However, less is known about sensorimotor changes required to face weightless environments when individuals have to perform fast and accurate goal-directed actions with whole-body displacement. We thus aimed at characterizing kinematic features of whole-body reaching movements in microgravity, involving high spatiotemporal constraints of execution, to question whether and how humans are able to maintain the performance of a functional behavior in the standards of normogravity execution. Seven participants were asked to reach as fast and as accurately as possible visual targets while standing during microgravity episodes in parabolic flight. Small and large targets were presented either close or far from the participants (requiring, in the latter case, additional whole-body displacement). Results reported that participants successfully performed the reaching task with general temporal features of movement (e.g., movement speed) close to land observations. However, our analyses also demonstrated substantial kinematic changes related to the temporal structure of focal movement and the postural strategy to successfully perform -constrained- whole-body reaching movements in microgravity. These immediate reorganizations are likely achieved by rapidly taking into account the absence of gravity in motor preparation and execution (presumably from cues about body limbs unweighting). Specifically, when compared to normogravity, the arm deceleration phase substantially increased. Furthermore, greater whole-body forward displacements due to smaller trunk flexions occurred when reaching far targets in microgravity. Remarkably, these changes of focal kinematics and postural strategy appear close to those previously reported when participants performed the same task underwater with neutral buoyancy applied to body limbs. Overall, these novel 
findings reveal that humans are able to maintain the performance of functional goal-directed whole-body actions in weightlessness by successfully managing spatiotemporal constraints of execution in this unusual environment.

Keywords: whole-body reaching, arm kinematics, postural strategy, sensorimotor adaptation, microgravity, parabolic flight, weightlessness

\section{INTRODUCTION}

On Earth, humans' motor behavior takes place within the ubiquitous gravitational force field. Several previous work already reported that the gravity direction and intensity are taken into account for motor execution, both on focal and postural components. For instance, regarding vertical arm movements, kinematic differences have been revealed between upward and downward movements (i.e., executed against or toward the direction of gravity). Particularly for upward arm movements, the relative deceleration duration was shown to be longer than the relative acceleration duration, while the opposite was observed for downward arm movements (Papaxanthis et al., 1998, 2003). Such asymmetric bell-shaped velocity profiles would allow humans to take advantage of mechanical effects of gravity torque on the limb by passively decelerating/accelerating upward/downward movements (Gaveau et al., 2014). This assumption is supported by the analysis of muscle activation patterns during vertical arm movements (Papaxanthis et al., 2003) and the removal of this specific asymmetry for horizontal movements wherein the gravitational torques did not vary (Gentili et al., 2007; Le Seac'h and McIntyre, 2007). Furthermore, these direction-dependent kinematic asymmetries appeared early in movement execution suggesting that the gravity effects could be anticipated and integrated into motor planning (Gaveau and Papaxanthis, 2011). Noticeably, the focal part of the movement investigated by these previous work is executed within a postural context, which was also subject to the influence of gravity. On Earth, body posture has to deal with the gravitational force to avoid falling. Indeed, humans would try to actively maintain the vertical projection of the center of mass (CoM) inside the support surface (Massion, 1992; Vernazza et al., 1996; Massion et al., 2004). Thus, trunk bending or upper limb movements may act as internal sources of disturbance to equilibrium. To prevent both substantial CoM displacement and falling, compensatory displacements of hip and knee usually occur (Babinski, 1899; Crenna et al., 1987; Massion, 1992; Horak, 2006).

Overall, studies mentioned above clearly demonstrated that the gravitational force plays an important role into the motor planning and execution on Earth. More precisely, the velocity profiles of arm movements and the postural strategy seem to be relevant gravity-dependent kinematic markers of human motor behavior. What happens however when gravity is removed? Understanding the impact of weightlessness on human behavior is of critical importance for keeping efficient sensorimotor behavior during the forthcoming long-term space missions. Parabolic and space flights contexts are privileged by researchers to investigate the effects of microgravity exposure on motor control. Previous studies focusing on arm movements revealed that final accuracy decreased in microgravity as compared to normogravity observations (Bock et al., 1992; Fisk et al., 1993; Watt, 1997; Carriot et al., 2004; Bringoux et al., 2012) which is consistent with works on pointing movements into a new force field (Lackner and DiZio, 1994; Shadmehr and MussaIvaldi, 1994; Goodbody and Wolpert, 1998; Bourdin et al., 2001, 2006; Lefumat et al., 2015). However, the way microgravity exposure impacts kinematic features remains unclear. Indeed, some authors observed a reduction of movement speed (Ross, 1991; Berger et al., 1997; Mechtcheriakov et al., 2002; Carriot et al., 2004; Crevecoeur et al., 2010) whereas others reported no significant changes as compared to normogravity (Papaxanthis et al., 2005; Bringoux et al., 2012; Gaveau et al., 2016). More interestingly, contrasting findings have been also reported concerning gravity-dependent kinematic markers based on the temporal organization of focal movement and postural behavior. Indeed, some studies of arm vertical movements performed during parabolic flights showed either a progressive disappearance of asymmetric velocity profiles (Papaxanthis et al., 2005; Gaveau et al., 2016) or conversely an increase of the relative deceleration duration (Bringoux et al., 2012) with respect to normogravity. Regarding postural control in microgravity, most previous work demonstrated the persistence of a terrestrial strategy by stabilizing the CoM displacements during internal disturbance, such as trunk bending or arm and leg raising (Massion et al., 1993, 1997; Mouchnino et al., 1996; VernazzaMartin et al., 2000). However, during long-term exposure, Pedrocchi et al. $(2002,2005)$ reported significant shifts of CoM toward the moving leg on a same lateral lower limb raising task.

In these previous experiments, it should be noted that the focal and postural parts of movement were separately investigated, although both components are known to largely interact during functional motor behavior. Only few works have studied goaldirected whole-body reaching movements in microgravity and contradictory findings were reported. On the one hand, Patron et al. (2005) reported a decrease of the relative deceleration duration of arm movement associated to a stabilized CoM displacement in microgravity. On the other hand, Casellato et al. (2012) reported an invariance of the asymmetry of the hand velocity profile as compared to normogravity data, associated to a vertical CoM projection beyond the base of support. These discrepant findings may partly originate from inter-individual variability, as Casellato et al. (2016) recently observed different and highly variable behaviors regarding CoM stabilization on three astronauts onboard the ISS (long-term exposure). Most importantly, task-related concerns, especially target location, body limbs displacements and movement speed, could also explain these contradictory results. For instance, Patron et al. (2005) investigated postural influences on a reaching task toward 
targets close to the participant's feet with or without speed instructions, while Casellato et al. (2012) asked the participants to perform unconstrained forward hand movements toward targets located beyond arm's length. However, to the best of our knowledge, we are not aware of any study which has investigated goal-directed whole-body reaching movements requiring to be performed as fast and as accurate as possible in microgravity.

The present study thus aimed at characterizing kinematic features of goal-directed whole-body reaching movements in microgravity, involving high spatiotemporal constraints of execution, by comparing them to normogravity observations. The spatial requirements were defined in terms of target location and size, while the temporal requirements referred to the necessity of performing the movements as fast as possible within the accuracy constraints. To that aim, close versus far external visual targets were presented during microgravity episodes in parabolic flight. To reach far targets, additional whole-body displacement was required. For both targets, two different sizes of target area were presented. As indicated by studies mentioned above, task requirements must be accounted for when considering the impact of microgravity on motor behavior. Thus, the high spatiotemporal constraints of execution in the present study constitute a novel approach allowing us to investigate whole-body reaching movements through a more functional behavior in weightless environments, close to those performed by astronauts during their space missions. In other words, we question whether and how humans are able to maintain the performance of a functional behavior in the standards of normogravity execution. We predicted substantial changes of gravity-dependent kinematic markers reflecting the specific reorganizations of focal and postural components in microgravity as compared to normogravity.

\section{MATERIALS AND METHODS}

\section{Participants}

Seven right-handed (3 women and 4 men, mean age $=39$ \pm 6.9 years) participated in the experiment on a voluntary basis. They had no prior experience of microgravity exposure. As the present study is part of a scientific program studying human motor behavior in different force fields, participants were previously tested in normogravity and underwater for the same task as reported in Macaluso et al. (2016). None of the participants suffered from neuromuscular or sensory impairments, as confirmed by a medical examination prior to the experiment. Vision was normal or corrected by lenses. Before microgravity exposure, the participants received comfort medication (scopolamine) to avoid motion sickness. It has been demonstrated that its use for parabolic flights did not induce neuromuscular side-effects on sensorimotor control (Ritzmann et al., 2016). All the participants were naive as to the specific purpose of the experiment, which was authorized by the ANSM (French National Agency for Biomedical Security) and approved by the Committee for the Protection of Persons concerned (CPP). The participants gave their signed informed consent prior to the study in accordance with the Helsinki Convention.

\section{Experimental Setup}

Circular targets were presented in front of participants standing upright and maintained to the ground structure by means of foot-straps (Figure 1A). They had to press their right index finger on the start push-button positioned alongside their body. The height of the start push-button was adjusted to each participant's height for initial posture standardization. Circular targets were oriented along the frontal plane and were positioned relative to participants' anthropometric features. Close targets were located at shoulder's height (i.e., the height of the target center corresponded to the horizontal projection of the height of the acromioclavicular joint in the sagittal plane) at a distance corresponding to arm length, allowing the participants to reach these targets without trunk displacement. Far targets were located $25 \mathrm{~cm}$ away and $20 \mathrm{~cm}$ below the close targets: in that case, participants had to perform additional trunk displacement to reach these targets (Figure 1B). For both target locations, the diameter was also manipulated through Light-Emitting Diodes (LEDs) equally distributed to define two target sizes: Small targets $4 \mathrm{~cm}$ or Large targets $10 \mathrm{~cm}$ (Figure 1C). Therefore, in this experiment, combining location and size corresponded to the presentation of four targets: CS (Close-Small), CL (Close-Large), FS (Close-Small), FL (Far-Large). Switching targets on and off were achieved by a homemade software (Docometre $\odot$ ) piloting a real-time acquisition/control system running at $10 \mathrm{kHz}$ (ADwin-Gold ${ }^{\complement}$, Jäger, Lorsch, Germany).

Markers were positioned onto the participants' index, shoulder and hip. Markers position was recorded (i) in normogravity with a video motion capture system (LEDtype markers) composed of three cameras sampled at $60 \mathrm{~Hz}$ (resolution: $848 \times 480$ pixels); (ii) in microgravity by an optical motion capture system (infra-red active markers) at

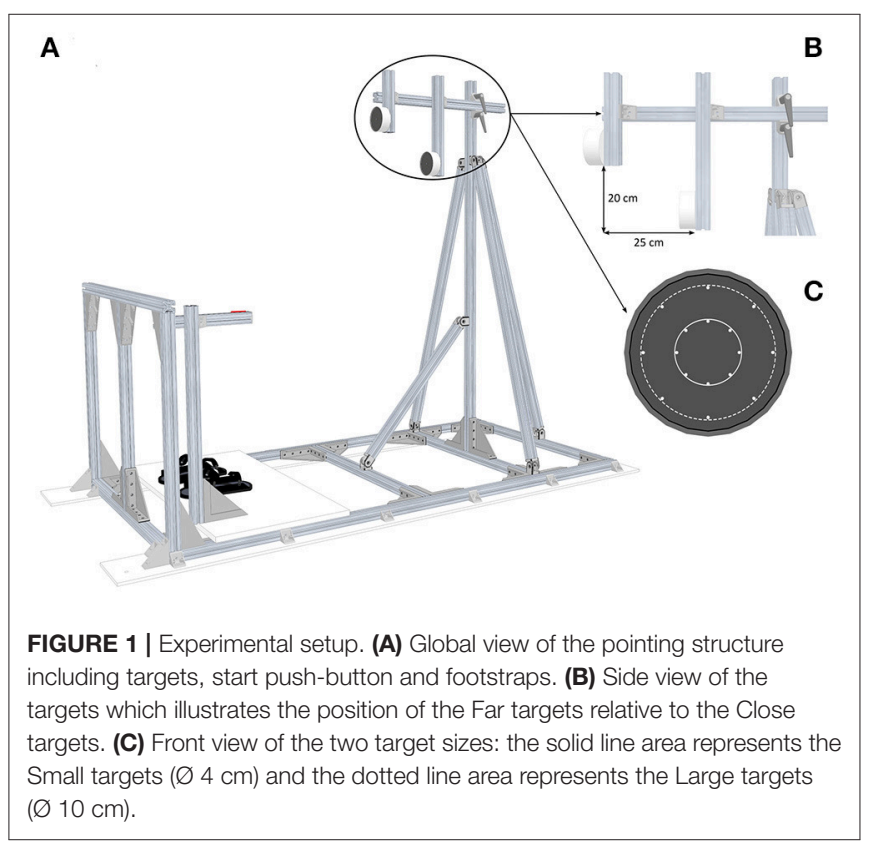


$200 \mathrm{~Hz}$ (Codamotion CXS and Active CodaHub, Charnwood Dynamics Ltd, Leicestershire, UK). Importantly, both acquisition systems yielded a similar accuracy in the definition of markers' position. Indeed, the data acquired in NormoG by the video motion capture system were processed using Direct Linear Transformation (Abdel-Aziz and Karara, 1971), to reach the accuracy level of the optical motion capture system used in MicroG (i.e., millimeter order). Moreover, according to the sampling theorem (Shannon, 1949), the sampling rates used in both environments are known to be sufficient to capture the whole range of velocities associated to biological motion, including fast reaching movements (Song and Godøy, 2016).

\section{Procedure}

All participants were exposed to two environments: first in normogravity ("NormoG") before the parabolic flight campaign, then in microgravity ("MicroG"). The MicroG environment was achieved in the A-310 ZERO-G aircraft chartered by the French Centre National d'Etudes Spatiales (CNES) and Novespace for parabolic flight studies, during the campaign \#125, including 3 days of flight. For each flight, the aircraft ran a sequence of 30 parabolas. Parabolic maneuver was composed of three distinct phases: $24 \mathrm{~s}$ of hypergravity $(1.8 \mathrm{~g}$, pull-up phase) followed by $22 \mathrm{~s}$ of microgravity $(0 \mathrm{~g})$ before a second period of $22 \mathrm{~s}$ of hypergravity (1.8 g, pull-out phase). Each parabola was separated by 1 min of normogravity (1 g, steady flight phase).

Positions of the start push-button and the targets were adjusted for each participant, then an initial calibration of targets was performed along the $\mathrm{Z}$ vertical axis (i.e., defining positions relative to arm movement elevation). Before each trial, participants had to stand upright, the arms outstretched along the body, and the right index pressing the start push-button. When one of the targets was illuminated, participants were asked to perform a reaching movement toward the target while keeping the arm outstretched. Reaching movements had to be performed as fast as possible while primarily respecting accuracy constraints related to the target area. Each trial was validated when the index fingertip reached the target. The final position had to be maintained until target extinction ( $3 \mathrm{~s}$ after movement onset) which prompted the participants to return to the starting position.

Participants performed 10 pointing movements toward each of the four targets for a total of 40 trials per experimental session in each environment. In the MicroG environment, these 40 trials were presented during 10 successive parabolas for each participant, thus including four trials per parabola. The targets were presented in a pseudorandom order, which was counterbalanced between the participants. Each session included three specific blocks of four trials in which the order of target presentation was the same. These blocks were presented in the initial, middle and final part of the session (corresponding to the 1, 5, and 10th parabola in MicroG) to assess the potential evolution of motor performance during each session, which lasted about $25 \mathrm{~min}$.

\section{Data Processing}

Data presented below describe behavioral features of reaching movements in the sagittal plane and some of them are detailed in Macaluso et al. (2016). First, we analyzed the fingertip trajectory, success rate (index fingertip within a given target area), index final deviation from target center, reaction time (RT), movement duration $(\mathrm{MD})$, and mean tangential velocity $\left(\mathrm{V}_{\text {mean }} \mathrm{endpoint}\right)$. The index final deviation was measured as the mean absolute distance of the final position of the index fingertip relative to the target center along the $\mathrm{Z}$ vertical axis. For each trial, the time elapsed between target illumination and the release of the start push-button by the participants defined RT. Index position in the sagittal plane was filtered (digital second-order dual-pass Butterworth filter; cutoff frequency $6 \mathrm{~Hz}$ in NormoG and $10 \mathrm{~Hz}$ in MicroG) and differentiated to obtain the endpoint tangential velocity in $\mathrm{m} . \mathrm{s}^{-1}$. Regarding the different sampling rates of acquisition systems used in both environments, we found that the cutoff frequencies mentioned above were the most suitable to reflect the raw data in normo and microgravity. The movement onset was defined as the time when the index tangential velocity reached $1.5 \%$ of its peak. Conversely, movement end was defined when the tangential velocity dropped below $1.5 \%$ of its peak.

The focal component of whole-body reaching movements was analyzed by considering the arm angular elevation over time (i.e., angle evolution of the extended arm around the shoulder with respect to its initial orientation). Arm angular elevation was computed from the index and shoulder XZ raw data, filtered (digital second-order dual-pass Butterworth filter; cutoff frequency $6 \mathrm{~Hz}$ in NormoG and $10 \mathrm{~Hz}$ in MicroG) and differentiated to obtain the arm angular velocity profile. From this velocity profile, the peak velocity $\left(\mathrm{PV}_{\mathrm{ang}}\right.$ in $\left.\mathrm{deg} . \mathrm{s}^{-1}\right)$ and the relative angular deceleration duration $\left(\mathrm{rDD}_{\mathrm{ang}}\right.$, defined as the duration between $\mathrm{PV}_{\mathrm{ang}}$ and movement end, expressed in $\%$ of movement duration to facilitate comparison between both environments) were extracted. Arm angular velocity profile was also differentiated to obtain arm angular acceleration profile, informing on early changes in motor execution which may give an insight upon the planning stage of focal movement. From this acceleration profile, peak acceleration $\left(\mathrm{PA}_{\mathrm{ang}}\right.$ in deg. $s^{-2}$ ) and time to peak acceleration $\left(\mathrm{TPA}_{\text {ang }}\right.$ expressed in $\mathrm{ms}$ to precisely estimate the occurrence of motor changes) were extracted.

In parallel, the postural component involved in the wholebody reaching movements (particularly to reach the far target) was analyzed by considering trunk displacement. This latter was illustrated by the final angular position of trunk (hip-shoulder segment) relative to vertical ( $\beta_{\mathrm{f}}$ trunk: trunk flexion in deg) at arm movement end, and by the forward displacement of participants' shoulder and hip (translation along the horizontal plane in $\mathrm{mm}$ ). Shoulder and hip movement onset/end in the sagittal plane were defined as the time when the tangential velocity respectively reached/dropped below $1.5 \%$ of its peak.

Statistical analyses were based on mean comparisons. Repeated-measures analyses of variance (ANOVAs) were performed to compare the means of kinematic parameters mentioned above after having ensured that the assumption of normality was not violated (Kolmogorov-Smirnov test). 
Newman-Keuls tests were used for post-hoc analyses and the significance threshold was set at.05 for all statistical tests.

\section{RESULTS}

\section{Potential Learning Effects}

We conducted prior analyses to investigate the potential learning effects during a single session (40 trials). Repeated-measures ANOVAs including 2 Environment (NormoG, MicroG) $\times 2$ Target Location (Close, Far) $\times 2$ Target Size (Small, Large) $\times 3$ Block (Initial, Middle, Final) were initially performed on all the selected parameters of the study. The results did not show any significant main effect of Block or any interaction with the other factors $(p>0.05)$. To specifically exclude the presence of any adaptive processes in MicroG environment, we conducted complementary analyses comparing a specific set of trials occurring during the 1, 5, and 10th parabola (see Material and Methods). Repeated-measures ANOVAs including 3 Parabola (1, 5, and 10th parabola) $\times 4$ Target Presentation (CS, CL, FS, FL) did not reveal any significant main effect of Parabola or any interaction with the other factor on all the selected parameters $(p>0.05)$. Thus, the reported values did not significantly change throughout the experiment.

\section{Upper-Limb Displacement}

First of all, we investigated arm movement toward the targets in each environment. Figure 2 illustrates mean endpoint trajectories (i.e., index fingertip) in the sagittal plane observed for a typical participant when reaching close and far targets. It shows that spatial characteristics of endpoint motion were impacted by the microgravity environment.

\section{Success Rate and Index Final Deviation}

Overall, participants successfully performed the task. Indeed, success rate was 100\% in NormoG and $95.42 \pm 8.99 \%$ in MicroG. In this latter environment, only the Small targets were sometimes missed (CS and FS). The ANOVA performed on success rate revealed no significant main effect of the experimental conditions (Environment: $p=0.06$; Target Location: $p=0.39$; Target Size: $p=0.06)$ and no significant interaction between these factors (Environment $\times$ Target Location: $p=0.39$; Environment $\times$ Target Size: $p=0.06$; Target Location $\times$ Target Size: $p=$ 0.39). Moreover, the ANOVA conducted on the index final deviation yielded no main effect of the experimental conditions (Environment: $p=0.10$; Target Location: $p=0.97$; Target Size: $p=0.06$ ) but showed a significant interaction between Environment $\times$ Target Size $\left[F_{(1,6)}=8.49 ; p<0.05\right]$. While no significant difference appeared between both environments when reaching Small targets $(p>0.05)$, the mean distance between the final position of the index and the target center when reaching Large targets was significantly higher in MicroG as compared to NormoG (13.04 $\pm 6.07 \mathrm{~mm}$ vs. $7.41 \pm 2.96 \mathrm{~mm} ; p<0.01)$. No significant interaction between the other factors was revealed (Environment $\times$ Target Location: $p=0.69$; Target Location $\times$ Target Size: $p=0.32$ ).

\section{Reaction Time (RT)}

The ANOVA performed on RT (mean $=326 \pm 70 \mathrm{~ms}$ ) revealed no significant main effect of the experimental conditions (Environment: $p=0.48$; Target Location: $p=0.23$; Target Size: $p=0.43$ ) and no significant interaction between these factors (Environment $\times$ Target Location: $p=0.19$; Environment $\times$ Target Size: $p=0.23$; Target Location $\times$ Target Size: $p=0.52$ ).

\section{Movement duration (MD) and Mean Tangential Velocity (Vmean endpoint $_{\text {) }}$}

The ANOVA conducted on MD only yielded a significant main effect of Target Location $\left[F_{(1,6)}=166.21 ; p<0.001\right]$; $\mathrm{MD}$ was longer when reaching Far targets $(0.73 \pm 0.17 \mathrm{~s})$ as compared to Close targets $(0.58 \pm 0.16 \mathrm{~s})$. No other significant main effect or interaction was found with regard to the other factors (Environment: $p=0.07$; Target Size: $p=$ 0.11; Environment $\times$ Target Location: $p=0.35$; Environment $\times$ Target Size: $p=0.26$; Target Location $\times$ Target Size: $p=0.59)$.

The ANOVA conducted on Vmean $_{\text {endpoint }}$ revealed significant main effects of Target Location $\left[F_{(1,6)}=24.05 ; p<0.01\right]$ and Target Size $\left[F_{(1,6)}=11.30 ; p<0.05\right]$. Vmean endpoint $_{\text {was higher }}$ when reaching Close targets $\left(1.94 \pm 0.39 \mathrm{~m} . \mathrm{s}^{-1}\right.$ vs. $1.66 \pm$ $0.31 \mathrm{~m} . \mathrm{s}^{-1}$, respectively for Close and Far targets). Vmean endpoint was also higher when reaching Large targets $\left(1.83 \pm 0.39 \mathrm{~m} . \mathrm{s}^{-1}\right.$ vs. $1.76 \pm 0.37 \mathrm{~m} . \mathrm{s}^{-1}$, respectively for Large and Small targets). No other significant main effect or interaction was found with regard to the other factors (Environment: $p=0.52$; Environment $\times$ Target Location: $p=0.14$; Environment $\times$ Target Size: $p=0.76$; Target Location $\times$ Target Size: $p=0.91$ ).

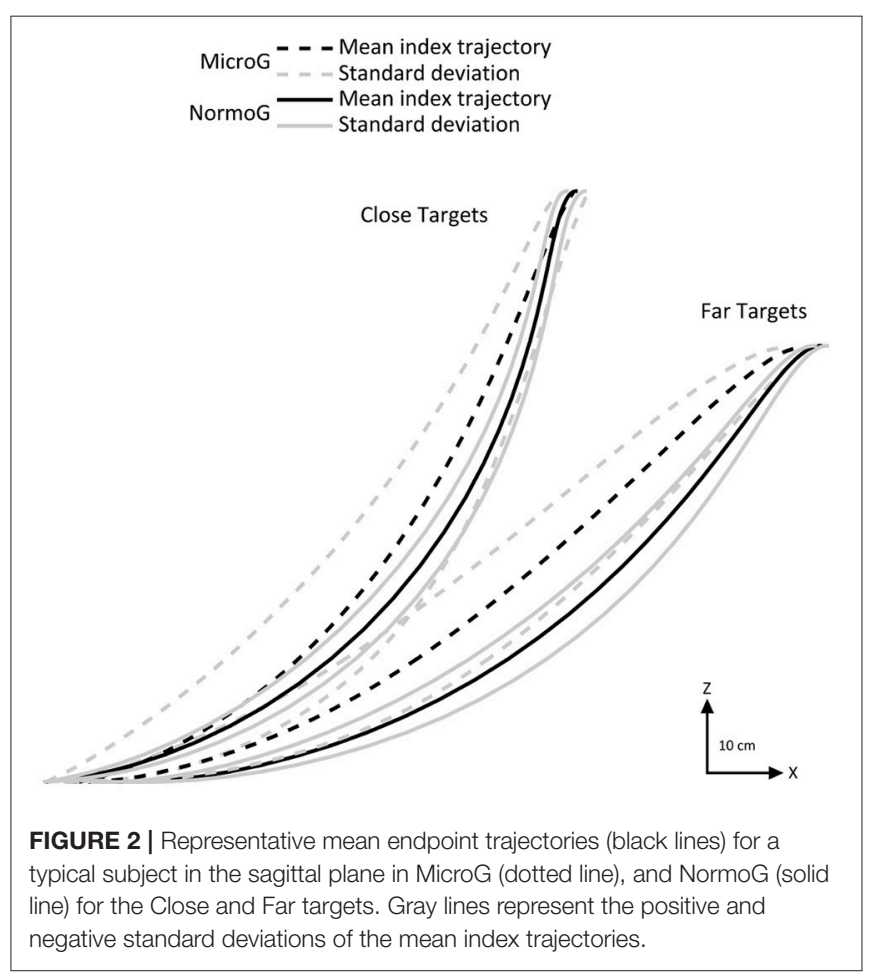


To sum up, microgravity did not significantly affect the performance of whole-body reaching movements without substantially disrupting the general temporal outputs of endpoint displacement and the success rate. Then, we investigated the temporal organization of the focal component illustrated by the arm angular elevation over time.

\section{Temporal Organization of Arm Angular Elevation}

Figure 3A illustrates mean arm angular velocity profiles for a typical participant when reaching Close and Far targets in each environment. It shows that the MicroG environment impacts the temporal structure of the velocity profile [reflected by the analysis of $r D D_{\text {ang }}$, see below Velocity profile: peak angular velocity $\left(\mathrm{PV}_{\text {ang }}\right)$ and relative angular deceleration duration $\left.\left(\mathrm{rDD}_{\mathrm{ang}}\right)\right]$ without substantially changing its amplitude. As reported below, this modulation could derive from changes of the temporal structure and amplitude of the acceleration profile [as suggested by the analysis of $T P A_{\text {ang }}$ and $P A_{\text {ang }}$, see below Acceleration profile: peak angular acceleration $\left(\mathrm{PA}_{\mathrm{ang}}\right)$ and time to peak angular acceleration $\left.\left(\mathrm{TPA}_{\mathrm{ang}}\right)\right]$.

\section{Velocity profile: peak angular velocity $\left(P V_{\text {ang }}\right)$ and relative} angular deceleration duration ( $\left.r D D_{a n g}\right)$

The ANOVA conducted on $\mathrm{PV}_{\text {ang }}$ only revealed a significant main effect of Target Location $\left[F_{(1,6)}=58.74 ; p<0.001\right]$. $\mathrm{PV}_{\text {ang }}$ was higher when reaching Close targets $\left(397.98 \pm 68.77\right.$ deg.s $\left.{ }^{-1}\right)$ as compared to Far targets $\left(327.55 \pm 48.39\right.$ deg. $\left.\mathrm{s}^{-1}\right)$. No other significant main effect or interaction was found with regard to the other factors (Environment: $p=0.29$; Environment $\times$ Target Location: $p=0.08$; Environment $\times$ Target Size: $p=0.73$; Target Location $\times$ Target Size: $p=0.51$ ).

The ANOVA conducted on $\mathrm{rDD}_{\text {ang }}$ revealed significant main effects of Environment $\left[F_{(1,6)}=48.54 ; p<0.001\right]$, Target Location $\left[F_{(1,6)}=20.91 ; p<0.01\right]$ and Target Size $\left[F_{(1,6)}\right.$ $=7.38 ; p<0.05]$. Importantly, $\mathrm{rDD}_{\mathrm{ang}}$ was substantially higher in MicroG as compared to NormoG (Figure 3B). Overall, $\mathrm{rDD}_{\text {ang }}$ was higher when reaching Far targets (69.65 $\pm 7.69 \% \mathrm{MD}$ vs. $60.80 \pm 7.63 \% \mathrm{MD}$, respectively for Far and Close targets) and Small targets too $(65.63 \pm 9.05 \% \mathrm{MD}$ vs. $64.83 \pm 8.71 \% \mathrm{MD}$, respectively for Small and Large targets). No significant interaction was found between these factors (Environment $\times$ Target Location: $p=0.22$; Environment $\times$ Target Size: $p=0.54$; Target Location $\times$ Target Size: $p=0.44$ ).

\section{Acceleration profile: peak angular acceleration $\left(P A_{a n g}\right)$ and time to peak angular acceleration (TPA ang}

The ANOVA performed on $\mathrm{PA}_{\text {ang }}$ revealed significant main effects of Environment $\left[F_{(1,6)}=9.30 ; p<0.05\right]$ and Target Location $\left[F_{(1,6)}=73.70 ; p<0.001\right]$. $\mathrm{PA}_{\text {ang }}$ was higher in MicroG than NormoG (Figure 3C) and also higher when reaching Close targets $\left(3661.18 \pm 1332.30\right.$ deg. $\left.\mathrm{s}^{-2}\right)$ as compared to Far targets $\left(3175.85 \pm 1265.36 \mathrm{deg} . \mathrm{s}^{-2}\right)$. No other significant main effect or interaction was found with regard to the other factors (Target Size: $p=0.54$; Environment $\times$ Target Location: $p=0.23$; Environment $\times$ Target Size: $p=0.99$; Target Location $\times$ Target Size: $p=0.98)$.
The ANOVA conducted on $\mathrm{TPA}_{\text {ang }}$ also yielded significant main effects of Environment $\left[F_{(1,6)}=7.43 ; p<0.05\right]$ and Target Location $\left[F_{(1,6)}=8.92 ; p<0.05\right)$. Importantly, $\mathrm{TPA}_{\text {ang }}$ was lower in MicroG than in NormoG (Figure 3D) and also lower when reaching Far targets ( $54 \pm 18 \mathrm{~ms})$ as compared to Close targets $(62 \pm 20 \mathrm{~ms})$. No other significant main effect or interaction was found with regard to the other factors (Target Size: $p=0.06$; Environment $\times$ Target Location: $p=0.92$; Environment $\times$ Target Size: $p=0.42$; Target Location $\times$ Target Size: $p=0.95$ ).

In summary, microgravity exposure influenced the temporal structure of arm angular elevation by decreasing the time to peak acceleration, thus leading to an increase of the relative deceleration duration as compared to NormoG. These modifications did not affect the maximal velocity of arm elevation in MicroG as compared to NormoG, presumably because of a higher maximal acceleration reached earlier during movement execution. The next part will focus on the postural component involved in whole-body reaching movements, particularly when reaching Far targets.

\section{Trunk Displacement Final Angular Position of Trunk Relative to Vertical $\left(\beta_{\mathrm{f}}\right.$ trunk)}

The ANOVA performed on $\beta_{\mathrm{f}}$ trunk revealed a main effect of Target Location $\left[F_{(1,6)}=264.09 ; p<0.001\right]$ and a significant interaction between Environment $\times$ Target Location $\left[F_{(1,6)}=\right.$ 24.74; $p<0.01$ ]. Interestingly, while no significant difference appeared between both environments when reaching Close targets $(p>0.05)$, mean $\beta_{\mathrm{f}}$ trunk was significantly lower when reaching Far targets in MicroG as compared to NormoG $(p<0.001$; Figure 4).

\section{Shoulder and Hip Forward Displacement}

Unsurprisingly in both environments, no noticeable forward translation was detected for shoulder and hip when reaching Close targets (located at participants' arm length, see Material and Methods). Therefore, we subsequently led our analysis on the shoulder and hip forward displacement occurring when reaching Far targets.

The ANOVA conducted on shoulder displacement yielded a significant main effect of Environment $\left[F_{(1,6)}=183.78\right.$; $p<0.001]$. Shoulder displacement in MicroG (448.28 \pm $25.37 \mathrm{~mm})$ was significantly higher than in NormoG (285.54 $\pm 36.18 \mathrm{~mm})$. The ANOVA performed on hip displacement revealed significant main effects of Environment $\left[F_{(1,6)}=20.94\right.$; $p<0.01]$ with higher displacement in MicroG $(185 \pm 84.28 \mathrm{~mm})$ as compared to NormoG $(38.68 \pm 39.49 \mathrm{~mm})$. The ANOVA also revealed a main effect of Target Size $\left[F_{(1,6)}=9.09 ; p<0.05\right]$ and a significant interaction between Environment $\times$ Target Size $\left[F_{(1,6)}=7.34 ; p<0.05\right]$. While no significant difference appeared between Small and Large targets in NormoG, mean hip displacement in MicroG was higher when reaching Large target $(191.66 \pm 86.15 \mathrm{~mm})$ as compared to Small target (178.50 $\pm 88.70 \mathrm{~mm})$.

Overall, these analyses highlight that the postural component varied during whole-body reaching movements mainly as a function of the Environment and Target Location. In MicroG, 


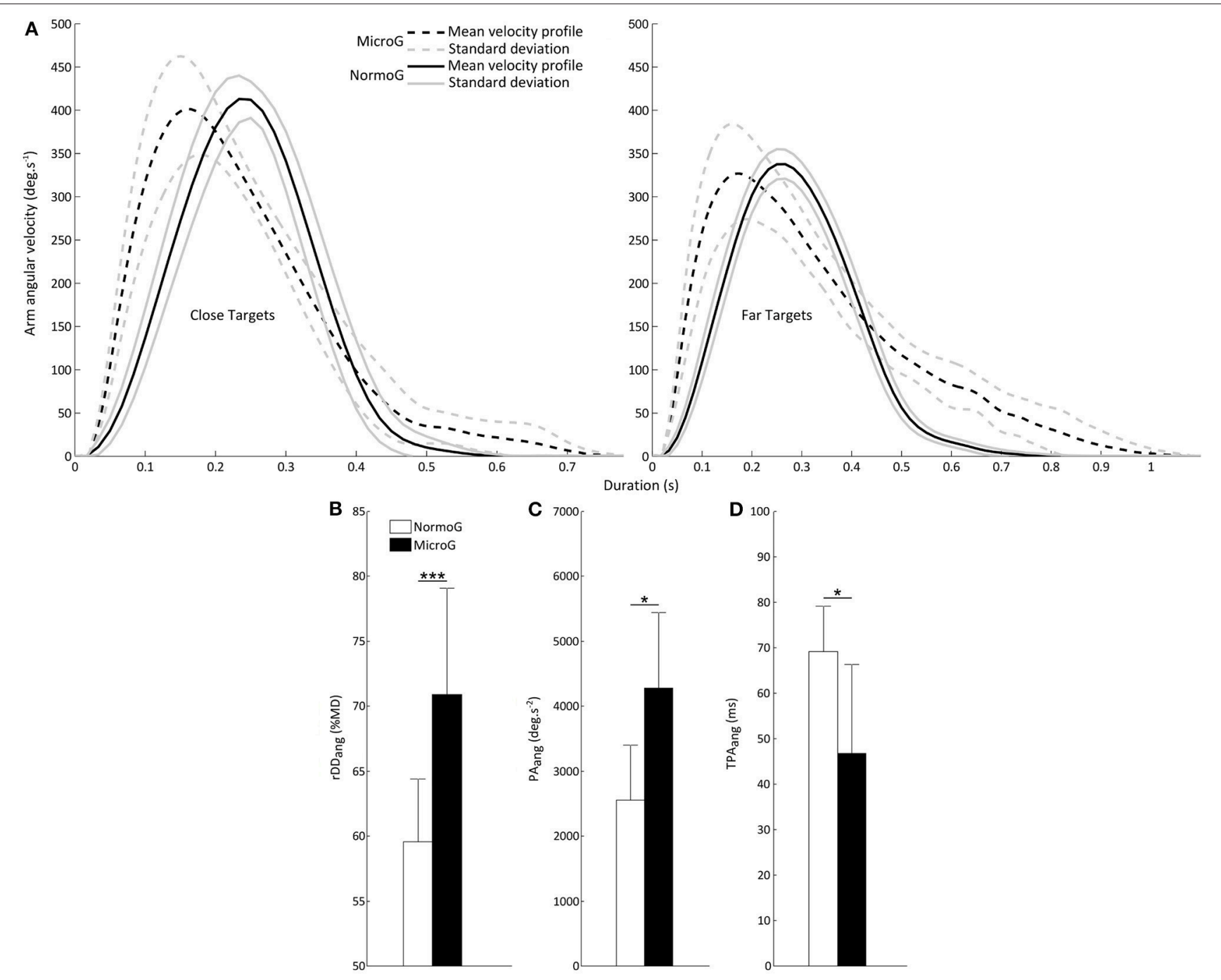

FIGURE 3 | (A) Representative mean arm angular velocity profiles for a typical subject in MicroG (dotted line) and NormoG (solid line) for the Close and Far targets. Gray lines represent the positive and negative standard deviations of the mean arm angular velocities. (B) Mean relative angular deceleration duration (rDDang) as a function of Environment. (C) Mean peak angular acceleration (PAang) and (D) Mean time to peak angular acceleration (TPAang) as a function of Environment. Error bars represent standard deviation of the mean. ${ }^{* *} p<0.001 ;{ }^{*} p<0.05$.

reaching Far targets involved smaller trunk bending associated to larger forward displacements of the shoulder and hip, as compared to NormoG. In the next section, we will discuss the main focal and postural features reported above and will propose possible interpretations for these observations.

\section{DISCUSSION}

The present study aimed at characterizing kinematic features of goal-directed whole-body reaching movements in microgravity involving high spatiotemporal constraints of execution, with respect to normogravity observations. Our original experimental design enabled us to investigate reaching movements performed as fast as possible toward targets of different sizes and locations in both environments. Our data revealed stabilized motor features throughout microgravity exposure. While some of them are associated to the preservation of general temporal outputs with respect to land observations (e.g., movement speed), we found substantial changes in gravity-dependent kinematic markers reflecting the reorganization of focal and postural components. These points will be developed in the following sections.

\section{Prompt Reorganization of Motor Behavior in Microgravity}

Although the participants never experienced microgravity exposure before the present experiment, we did not find any significant evolution in the reported variables across the successive trials. Thus, we failed to show the presence of sensorimotor adaptation during the experiment which would indeed have led to more progressive changes across 


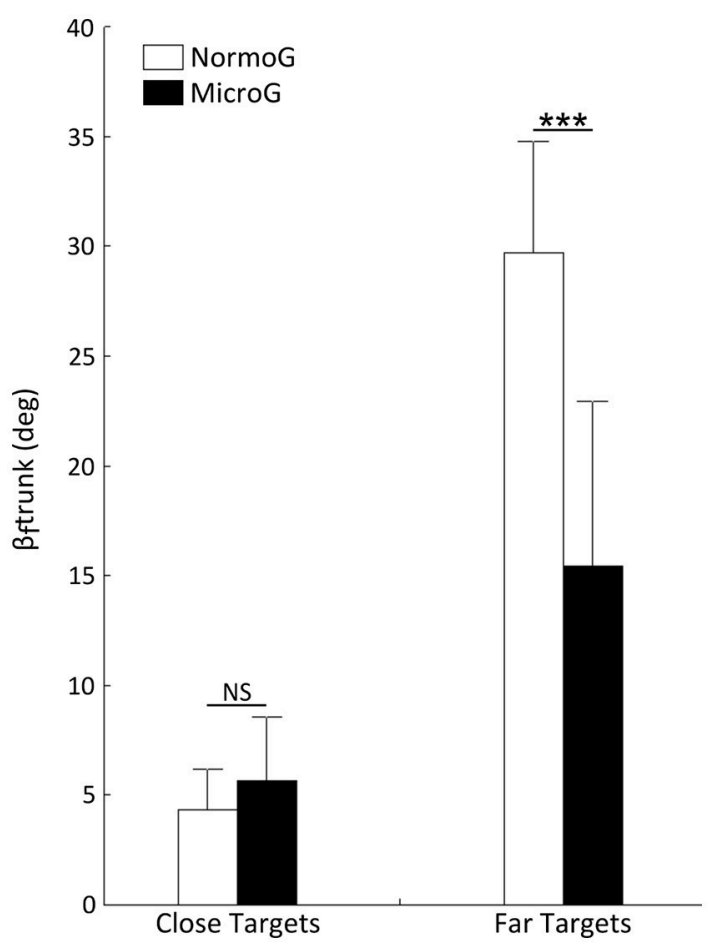

FIGURE 4 | Mean final angular position of trunk relative to vertical ( $\beta_{\mathrm{f}}$ trunk) as a function of Environment and Target Location. Error bars represent standard deviation of the mean. ${ }^{\star \star \star} p<0.001$; NS, non-significant difference.

the repetition of reaching movements (Lackner and DiZio, 1994; Shadmehr and Mussa-Ivaldi, 1994). Rather, we observed a prompt reorganization of some movement features (see section Microgravity Is Accounted for into the Planning of Focal Movement) which took place at the earliest onset of exposure. From previous work conducted in parabolic flights, the occurrence of adaptive processes on reaching movements is not clear. Indeed, some studies reported slow progressive changes of kinematics across parabolas (Papaxanthis et al., 2005; Gaveau et al., 2016) whereas others observed rapid behavioral stabilization or no significant change during exposure (Patron et al., 2005; Bringoux et al., 2012; Casellato et al., 2012). In our study, one hypothesis related to the parabolic flight context can be advanced to explain this immediate stabilization of motor behavior. Before the 30 parabolas achieved for experimental acquisition, the aircraft performed one parabola to allow participants discover the parabolic maneuver. Moreover, since 3 participants were tested during each flight (see Material and Methods), two of them had even more time to experience microgravity exposure. Although we ensured that no reaching movements were performed by the participants out of the experiment, this preliminary although short exposure before data acquisition would enable the participants to develop prior expectancies about how it feels to move in these novel environments. Moreover, microgravity episodes of parabolic flights induced a global modification of the force field applied to the whole-body before initiating each trial. Thus, in this context, the participants accessed the new dynamic properties of the environment prior to movement onset (Barbiero et al., 2017) which might be sufficient to rapidly update their internal model for sensorimotor planning and execution, hence leading to an immediate motor reorganization (Wolpert and Kawato, 1998; Wolpert and Ghahramani, 2000). It has been shown indeed that the initial state of the sensorimotor system is primarily used to adjust the internal representations necessary to perform upcoming movements (Starkes et al., 2002; Flanagan et al., 2006; White et al., 2012, Rousseau et al., 2016). Here, the limb proprioception could contribute to detect the gravity release at the level of muscles and joints, through muscle spindles and Golgi tendon organs identified as load receptors related to gravity force field (Dietz et al., 1992). The following sections aim at discussing the stabilized motor features observed in microgravity for whole-body reaching.

\section{Preservation of Functional Reaching Movements within Normogravity Standards}

The high spatiotemporal constraints of execution in the present study enabled us to investigate functional whole-body reaching movements in microgravity. In this line, our data did not reveal any significant difference between MicroG and NormoG environments in terms of movement duration, mean and peak velocity during movement execution. The absence of effect of the environment on these variables may reflect a tendency to keep the average movement speed in the range of normogravity experience. To that aim, the participants may have reduced the safety margin related to the final reaching accuracy in microgravity. Indeed, while the preservation of movement speed was not detrimental to reaching performance (i.e., the high success rate observed in MicroG, > 95\%, was not significantly different from NormoG), the distance between the endpoint final position and the target center was significantly higher in MicroG when reaching large targets. Thus, in this task, the participants tended to maintain speed over accuracy margin (Woodworth, 1899) for a still successful performance without gravity. Keeping the average speed and reaching performance within normogravity standards were though not at the expense of movement preparation duration, since the reaction time remained also unaffected by the environment. Hence, alleviating gravity before movement execution did not impact the time allocated for motor planning. Nevertheless, we will detail in the following parts some evidence for substantial qualitative reorganizations, notably in focal and postural components of the reaching movement, which helped maintain the functionality of motor behavior in microgravity.

\section{Microgravity Is Accounted for into the Planning of Focal Movement}

On Earth, kinematics of arm movement elevation has been welldescribed in terms of asymmetric bell-shaped velocity profiles (Papaxanthis et al., 1998; Gentili et al., 2007). Classically, the relative deceleration duration appears longer than the relative acceleration duration, suggesting that gravity is accounted for 
during motor planning to act as an assistive force for decelerating upward movements (Papaxanthis et al., 2003; Gaveau and Papaxanthis, 2011). The way gravity is integrated into motor planning has been recently formalized by a Minimum SmoothEffort model (Gaveau et al., 2016), in line with the optimal control theory minimizing absolute work and jerk (Berret et al., 2011; Gaveau et al., 2014).

In our experiment, the substantial increase of the relative deceleration duration in MicroG constitutes the most salient feature of motor reorganization concerning the focal part of the reaching movement. The asymmetry was thus notably amplified in microgravity without changing the amplitude of peak velocity. Such reorganization, consistent across subjects as shown in the Supplementary Figure 1A, appears as a direct consequence of an earlier peak acceleration observed during motor execution ( $\sim 47 \mathrm{~ms})$. Recent work established that the shortest feedbackbased corrections of EMG (electromyographic) patterns during arm reaching occur at $\sim 60 \mathrm{~ms}$ when considering a limb disturbance with no changes of target location (Scott, 2016 for a review). We therefore hypothesize that the kinematic changes promptly observed in microgravity following arm movement onset are based on feedforward control mechanisms, directly expressed in the motor intention (Gaveau and Papaxanthis, 2011). In other words, we argue that the CNS could predict the effect of gravity release on the moving segments and could subsequently integrate the novel dynamics associated to a weightless environment into motor planning. As there is no external force to help braking upward movements in microgravity (neglecting the air friction forces), the participants had to actively counteract the inertial force of their moving limbs, presumably by increasing the antagonist muscle activations (Bonnard et al., 1997). In this context, a longer deceleration phase may reflect a greater use of feedback processes (Chua and Elliott, 1993; Sarlegna et al., 2003; Terrier et al., 2011). This greater retroactive control would enable the participants to better manage the speed reduction of their reaching movements, especially when approaching the target, to maintain final accuracy. Increased asymmetry between acceleration and deceleration phases was also reported when removing gravitational shoulder torque before arm movement onset (Rousseau et al., 2016) and with additional loads placed on the arm (Gaveau et al., 2011). Such reorganization in kinematics may thus illustrate a cautious strategy accounting for force/inertia uncertainties in unusual context. In this line, previous studies demonstrated that the lack of information prior to movement onset strongly affects motor planning (Bringoux et al., 2012; Rousseau et al., 2016), presumably to face unexpected or erroneous sensorimotor estimates during subsequent movement execution in unfamiliar environments (Brooks et al., 2015).

Contradictory with the present findings, some other studies reported a progressive disappearance of asymmetric velocity profiles in microgravity (Papaxanthis et al., 2005; Gaveau et al., 2016). Unlike our experiment, movement accuracy was not a primary constraint in these previous work where the braking phase of arm movements was not crucial during motor execution to correctly perform the task. Alternatively, when the participants had to perform reaching movements "as accurately as possible" in microgravity, Bringoux et al. (2012) also observed a longer deceleration phase as compared to normogravity exposure. Interestingly in our study, such reorganization of motor planning for arm reaching in MicroG was not detrimental to movement duration: the longer deceleration phase was compensated by higher peak acceleration in microgravity. This compensatory increase of peak acceleration may also represent a specific reorganization of the movement in a given environment as it likely exploits the absence of gravity torque at movement onset to efficiently trigger the initial impulse. Additionally, as discussed in the following section, the planning of focal movement was not the only component to be modified during whole-body reaching movements in microgravity.

\section{Efficient Postural Strategy for Reaching Without Gravity}

Kinematics collected from the trunk clearly supports two different postural strategies as a function of the gravity environment while reaching far targets placed beyond arm's length. Under normogravity, our analyses revealed a significant forward trunk bending expressed by large shoulder displacement associated to very small hip displacement in space. This feature is typical of a "hip strategy" (Horak and Nashner, 1986), through which the postural component supporting the focal part of movement is also used to prevent falling (Massion, 1992). This posturokinetic organization would thus reduce the displacement of the CoM by using compensatory mechanisms (Massion, 1992; Vernazza et al., 1996) and would favor equilibrium maintenance at the expense of mechanical energy minimization and joint smoothness maximization (Hilt et al., 2016).

Alternatively, the second postural strategy specifically observed in microgravity was illustrated by very small trunk bending associated to larger shoulder and hip displacement from vertical. This organization would resemble the "ankle strategy" evoked by Horak and Nashner (1986), though with greater whole-body forward displacement. In MicroG environment, the participants were indeed not constrained by gravitational force, allowing for a vertical CoM projection outside the base of support. This observation is consistent with others reporting that postural control in weightlessness is predominantly managed at the ankle level (Clement et al., 1984; Clément and Lestienne, 1988). On Earth, this posturokinetic strategy decreases the equilibrium safety margin but the risk of falling is greatly minimized in microgravity. The participants might therefore adopt the strategy which would allow them to reduce the degrees of freedom (Bernstein, 1967), helping minimize the mechanical energy expenditure and maximize joint smoothness (Hilt et al., 2016). In line with the optimal control theory (Berret et al., 2011; Gaveau et al., 2014), the combination of these cost functions would enable the postural component to support more efficiently the focal part of the reaching movement in weightless environment. Despite methodological differences with our study, Casellato et al. (2012) also reported whole-body forward displacement when performing unconstrained bimanual reaching (i.e., natural pace and uncontrolled accuracy). In line with their observations, our 
data may also support the existence of an "oversimplification" of postural control to perform a functional behavior when facing high spatiotemporal constraints of execution in microgravity. Moreover, unlike previous observations of Casellato et al. (2016) in long-term weightlessness, the postural strategy observed in the present study was not subjected to large inter-individual variability. Overall, individual trends for both focal and postural observations are clear and systematic (as illustrated in the Supplementary Figure 1). Remarkably, the stabilized motor features observed in microgravity in the present study appear close to those previously reported when participants performed the same task underwater with neutral buoyancy applied to body limbs (Macaluso et al., 2016). The following section discusses the behavioral analogies observed in both environments.

\section{Behavioral Analogies between Neutral Buoyancy Underwater and Microgravity}

As the present study is part of a scientific program studying human motor behavior in different force fields, the same participants were previously tested underwater in the same task for comparison purpose. Specifically, they were immersed in a prototypical submersible simulated space suit (AquaS environment; Macaluso et al., 2016), to apply neutral buoyancy at the level of body limbs. As in the present study conducted 2 years later, we did not find any significant evolution in the reported variables across the successive trials performed underwater. Rather, we observed immediate reorganizations at the earliest onset of exposure excluding the presence of adaptive processes during the experiment. As in MicroG, AquaS environment also implied initial exposure before data acquisition related to the installation of participants on the pointing structure. Thus, participants were submitted to global modifications of the force field applied to the whole-body before trial execution. This observation extends the hypothesis provided in section Prompt Reorganization of Motor Behavior in Microgravity. When participants accessed the new "unweighting" properties of a given environment before performing the first reaching movements, they could promptly reorganize their motor behavior. Most interestingly, the changes of focal and postural components of reaching movements in MicroG are close to those observed underwater in AquaS. Indeed, the increase of the relative arm deceleration duration and the decrease of trunk flexion when reaching far targets appear strikingly comparable (see the Supplementary Figure 2). In other words, the participants adopted analogous temporal structure of arm movements and almost similar postural strategy to perform whole-body reaching movements in these different environments. In so far as these two parameters are known to be gravity-dependent kinematic markers (see Introduction), and as AquaS and MicroG environments attempted to reproduce a weightless context, we hypothesize that these very close motor strategies would be mainly due to whole-body unweighting. It suggests that a fine control of neutral buoyancy underwater across the whole-body segments would tend to better simulate microgravity when considering the execution of sensorimotor tasks. Further studies are obviously required to challenge this hypothesis, especially to better investigate the effects of viscous force on motor control.

\section{CONCLUSION}

The present study provides clear and original evidence that participants could successfully perform goal-directed wholebody reaching movements involving high spatiotemporal constraints in a novel environment, such as microgravity, by immediately reorganizing focal and postural control strategies compared to normogravity. Moreover, these substantial modifications occurred in motor planning at the very beginning of weightless exposure which strongly suggests that the effects of the absence of gravity were anticipated and integrated by CNS. Overall, our novel findings highlight that humans are able to maintain the performance of functional goaldirected whole-body actions in weightlessness in the standards of normogravity observations by successfully managing spatiotemporal constraints of execution in this unusual environment. Interestingly, our previous work reported similar kinematic features of whole-body reaching movements performed underwater when neutral buoyancy was rigorously applied at the level of each body limb (Macaluso et al., 2016). Therefore, this suggests that comparable initial state estimates and subsequent motor reorganizations could arise from unweighting the body at the level of body skin, muscles and joints, irrespective of the presence of gravity-related vestibular cues. Further experiments are of course mandatory to investigate this challenging hypothesis, which may be crucial for instance in astronauts training underwater, where gravitational field still acts at the level of the vestibular system.

\section{ETHICS STATEMENT}

This study was carried out in accordance with the recommendations of the ANSM (French National Agency for Biomedical Security) with written informed consent from all subjects. All subjects gave written informed consent in accordance with the Declaration of Helsinki. The protocol was approved by the CPP.

\section{AUTHOR CONTRIBUTIONS}

TM designed and performed experiments, analyzed data and wrote the paper; $\mathrm{CB}$ wrote the paper; $\mathrm{FB}$ designed experiments and analyzed data; MM wrote the paper; PS designed and performed experiments, analyzed data; FS wrote the paper; JV wrote the paper; LB designed and performed experiments, wrote the paper.

\section{FUNDING}

This work (SIMEXPLOR) was supported by the Centre National d'Etudes Spatiales (CNES) through the APR Grants and the CNRS (Centre National de la Recherche Scientifique). 
The funders had no role in study design, data collection and analysis, decision to publish, or preparation of the manuscript.

\section{ACKNOWLEDGMENTS}

The authors wish to thank Novespace and CADMOS (CNES) for technical support, Patrick Sandor for medical inclusion of

\section{REFERENCES}

Abdel-Aziz, Y. I., and Karara, H. M. (1971). Direct linear transformation from comparator coordinates into object space coordinates. Am. Soc. Photogramm. $40,1-18$.

Babinski, J. (1899). De l'asynergie cérébelleuse. Rev. Neurol. 7, 806-816.

Barbiero, M., Rousseau, C., Papaxanthis, C., and White, O. (2017). Coherent multimodal sensory information allows switching between gravitoinertial contexts. Front. Physiol. 8:290. doi: 10.3389/fphys.2017.00290

Berger, M., Mescheriakov, S., Molokanova, E., Lechner, S. S., Seguer, N., and Kozlovskaya, I. (1997). Pointing arm movements in short- and long-term spaceflights. Aviat. Space Environ. Med. 68, 781-787.

Bernstein, N. A. (1967). The Co-ordination and Regulation of Movements. Oxford: Pergamon Press.

Berret, B., Chiovetto, E., Nori, F., and Pozzo, T. (2011). Evidence for composite cost functions in arm movement planning: an inverse optimal control approach. PLoS Comput. Biol. 7:e1002183. doi: 10.1371/journal.pcbi.1002183

Bock, O., Howard, I. P., Money, K. E., and Arnold, K. E. (1992). Accuracy of aimed arm movements in changed gravity. Aviat. Space Environ. Med. 63, 994-998.

Bonnard, M., Pailhous, J., and Danion, F. (1997). Intentional on-line adaptation of rhythmic movements during a hyper- to microgravity change. Motor Control. 1, 247-262. doi: 10.1123/mcj.1.3.247

Bourdin, C., Bringoux, L., Gauthier, G. M., and Vercher, J.-L. (2006). Vision of the hand prior to movement onset allows full motor adaptation to a multi-force environment. Brain Res. Bull. 71, 101-110. doi: 10.1016/j.brainresbull.2006.08.007

Bourdin, C., Gauthier, G. M., Blouin, J., and Vercher, J.-L. (2001). Visual feedback of the moving arm allows complete adaptation of pointing movements to centrifugal and Coriolis forces. Neurosci. Lett. 301, 25-28. doi: 10.1016/S0304-3940(01)01584-1

Bringoux, L., Blouin, J., Coyle, T., Ruget, H., and Mouchnino, L. (2012). Effect of gravity like torque on goal-directed arm movements in microgravity. J. Neurophysiol. 107, 2541-2548. doi: 10.1152/jn.00364.2011

Brooks, J. X., Carriot, J., and Cullen, K. E. (2015). Learning to expect the unexpected: rapid updating in primate cerebellum during voluntary selfmotion. Nat. Neurosci. 18, 1310-1317. doi: 10.1038/nn.4077

Carriot, J., Bringoux, L., Charles, C., Mars, F., Nougier, V., and Cian, C. (2004). Perceived body orientation in microgravity: effects of prior experience and pressure under the feet. Aviat. Space Environ. Med. 75, 795-799.

Casellato, C., Pedrocchi, A., and Ferrigno, G. (2016). Whole-body movements in long-term weightlessness: hierarchies of the controlled variables are gravity-dependent. J. Mot. Behav. 49, 568-579. doi: 10.1080/00222895.2016.12 47032

Casellato, C., Tagliabue, M., Pedrocchi, A., Papaxanthis, C., Ferrigno, G., and Pozzo, T. (2012). Reaching while standing in microgravity: a new postural solution to oversimplify movement control. Exp. Brain Res. 216, 203-215. doi: 10.1007/s00221-011-2918-2

Chua, R., and Elliott, D. (1993). Visual regulation of manual aiming. Hum. Mov. Sci. 12, 365-401. doi: 10.1016/0167-9457(93)90026-L

Clement, G., Gurfinkel, V. S., Lestienne, F., Lipshits, M. I., and Popov, K. E. (1984). Adaptation of postural control to weightlessness. Exp. Brain Res. 57, 61-72. doi: 10.1007/BF00231132

Clément, G., and Lestienne, F. (1988). Adaptive modifications of postural attitude in conditions of weightlessness. Exp. Brain Res. 72, 381-389. doi: $10.1007 /$ BF00250259 the participants, Jean-François Bramard and Alexis Rosenfeld for video assistance and the participants who took part in this study.

\section{SUPPLEMENTARY MATERIAL}

The Supplementary Material for this article can be found online at: https://www.frontiersin.org/articles/10.3389/fphys. 2017.00821/full\#supplementary-material

Crenna, P., Frigo, C., Massion, J., and Pedotti, A. (1987). Forward and backward axial synergies in man. Expl. Brain Res. 65, 538-548. doi: 10.1007/BF00235977

Crevecoeur, F., McIntyre, J., Thonnard, J.-L., and Lefèvre, P. (2010). Movement stability under uncertain internal models of dynamics. J. Neurophysiol. 104, 1301-1313. doi: 10.1152/jn.00315.2010

Dietz, V., Gollhofer, A., Kleiber, M., and Trippel, M. (1992). Regulation of bipedal stance: dependency on "load" receptors. Exp. Brain Res. 89, 229-231. doi: $10.1007 / \mathrm{BF} 00229020$

Fisk, J., Lackner, J. R., and DiZio, P. (1993). Gravitoinertial force level influences arm movement control. J. Neurophysiol. 69, 504-511.

Flanagan, J. R., Bowman, M. C., and Johansson, R. S. (2006). Control strategies in object manipulation tasks. Curr. Opin. Neurobiol. 16, 650-659. doi: 10.1016/j.conb.2006.10.005

Gaveau, J., Berret, B., Angelaki, D. E., and Papaxanthis, C. (2016). Directiondependent arm kinematics reveal optimal integration of gravity cues. eLife 5:e16394. doi: 10.7554/eLife.16394

Gaveau, J., Berret, B., Demougeot, L., Fadiga, L., Pozzo, T., and Papaxanthis, C. (2014). Energy-related optimal control accounts for gravitational load: comparing shoulder, elbow, and wrist rotations. J. Neurophysiol. 111, 4-16. doi: 10.1152/jn.01029.2012

Gaveau, J., Paizis, C., Berret, B., Pozzo, T., and Papaxanthis, C. (2011). Sensorimotor adaptation of point-to-point arm movements after spaceflight: the role of internal representation of gravity force in trajectory planning. J. Neurophysiol. 106, 620-629. doi: 10.1152/jn.00081.2011

Gaveau, J., and Papaxanthis, C. (2011). The temporal structure of vertical arm movements. PLoS ONE 6:e22045. doi: 10.1371/journal.pone.0022045

Gentili, R. J., Cahouet, V., and Papaxanthis, C. (2007). Motor planning of arm movements is direction-dependent in the gravity field. Neuroscience 145, 20-32. doi: 10.1016/j.neuroscience.2006.11.035

Goodbody, S. J., and Wolpert, D. M. (1998). Temporal and amplitude generalization in motor learning. J. Neurophysiol. 79, 1825-1838.

Hilt, P. M., Berret, B., Papaxanthis, C., Stapley, P. J., and Pozzo, T. (2016). Evidence for subjective values guiding posture and movement coordination in a freeendpoint whole-body reaching task. Sci. Rep. 6:23868. doi: 10.1038/srep23868

Horak, F. B. (2006). Postural orientation and equilibrium: what do we need to know about neural control of balance to prevent falls? Age and Ageing. 35:S2 ii7-ii11. doi: 10.1093/ageing/afl077

Horak, F. B., and Nashner, L. M. (1986). Central programming of postural movements: adaptation to altered support-surface configurations. J. Neurophysiol. 55, 1369-1381.

Lackner, J. R., and DiZio, P. (1994). Rapid adaptation to coriolis force perturbations of arm trajectory. J. Neurophysiol. 72, 299-313.

Lefumat, H. Z., Vercher, J.-L., Miall, R. C., Cole, J., Buloup, F., Bringoux, L., et al. (2015). To transfer or not to transfer? Kinematics and laterality quotient predict interlimb transfer of motor learning. J. Neurophysiol. 5, 2764-2774. doi: 10.1152/jn.00749.2015

Le Seac'h, A. B., and McIntyre, J. (2007). Multimodal reference frame for the planning of vertical arms movements. Neurosci. Lett. 423, 211-215. doi: 10.1016/j.neulet.2007.07.034

Macaluso, T., Bourdin, C., Buloup, F., Mille, M.-L., Sainton, P., Sarlegna, F. R., et al. (2016). Kinematic features of whole-body reaching movements underwater: neutral buoyancy effects. Neuroscience 327, 125-135. doi: 10.1016/j.neuroscience.2016.04.014

Massion, J. (1992). Movement, posture and equilibrium: interaction and coordination. Prog. Neurobiol. 38, 35-56. doi: 10.1016/0301-0082(92)90034-C 
Massion, J., Alexandrov, A., and Frolov, A. (2004). Why and how are posture and movement coordinated? Prog. Brain Res. 143, 13-27. doi: 10.1016/S0079-6123(03)43002-1

Massion, J., Gurfinkel, V., Lipshits, M., Obadia, A., and Popov, K. (1993). Axial synergies under microgravity conditions. J. Vestib. Res. 3, 275-287.

Massion, J., Popov, K., Fabre, J. C., Rage, P., and Gurfinkel, V. (1997). Is the erect posture in microgravity based on the control of trunk orientation or center of mass position? Exp. Brain Res. 114, 384-389. doi: 10.1007/PL00005647

Mechtcheriakov, M., Berger, E., Molokanova, G., Holzmueller, W., Wirtenberger, S., Lechner-Steinleitner, C., et al. (2002). Slowing of human arm movements during weightlessness: the role of vision. Eur. J. Appl. Physiol. 87, 576-583. doi: 10.1007/s00421-002-0684-3

Mouchnino, L., Cincera, M., Fabre, J. C., Assaiante, C., Amblard, B., Pedotti, A., et al. (1996). Is the regulation of the center of mass maintained during leg movement under microgravity conditions? J. Neurophysiol. 76, 1212-1223.

Papaxanthis, C., Pozzo, T., and McIntyre, J. (2005). Kinematic and dynamic processes for the control of pointing movements in humans revealed by short-term exposure to microgravity. Neuroscience 135, 371-383. doi: 10.1016/j.neuroscience.2005.06.063

Papaxanthis, C., Pozzo, T., and Schieppati, M. (2003). Trajectories of arm pointing movements on the sagittal plane vary with both direction and speed. Exp. Brain Res. 148, 498-503. doi: 10.1007/s00221-002-1327-y

Papaxanthis, C., Pozzo, T., and Stapley, P. (1998). Effects of movement direction upon kinematic characteristics of vertical arm pointing movements in man. Neurosci. Lett. 253, 103-106. doi: 10.1016/S0304-3940(98)00604-1

Patron, J., Stapley, P., and Pozzo, T. (2005). Human whole-body reaching in normal gravity and microgravity reveals a strong temporal coordination between postural and focal task components. Exp. Brain Res. 165, 84-96. doi: 10.1007/s00221-005-2283-0

Pedrocchi, A., Baroni, G., Mouchnino, L., Ferrigno, G., Pedotti, A., and Massion, J. (2002). Absence of center of mass control for leg abduction in long- term weightlessness in humans. Neurosci. Lett. 319, 172-176. doi: 10.1016/S0304-3940(02)00002-2

Pedrocchi, A., Baroni, G., Pedotti, A., Massion, J., and Ferrigno, G. (2005). Inverse dynamic investigation of voluntary leg lateral movements in weightlessness: a new microgravity-specific strategy. J. Biomech. 38, 769-777. doi: 10.1016/j.jbiomech.2004.05.043

Ritzmann, R., Freyler, C., Krause, A., and Gollhofer, A. (2016). No neuromuscular side-effects of scopolamine in sensorimotor control and force-generating capacity among parabolic fliers. Microgr. Sci. Technol. 28, 477-490. doi: $10.1007 /$ s12217-016-9504-y

Ross, H. E. (1991). Motor skills under varied gravitoinertial force in parabolic flight. Acta Astro. 23, 85-89. doi: 10.1016/0094-5765(91)90103-C

Rousseau, C., Papaxanthis, C., Gaveau, J., Pozzo, T., and White, O. (2016). Initial information prior to movement onset influences kinematics of upward arm pointing movements. J. Neurophysiol. 116, 1673-1683. doi: 10.1152/jn.00616.2015

Sarlegna, F., Blouin, J., Bresciani, J.-P., Bourdin, C., Vercher, J.-L., and Gauthier, G. M. (2003). Target and hand position information in the online control of goal-directed arm movements. Exp. Brain. Res. 151, 524-535. doi: 10.1007/s00221-003-1504-7

Scott, S. H. (2016). A functional taxonomy of bottom-up sensory feedback processing for motor actions. Trends Neurosci. 39, 512-526. doi: 10.1016/j.tins.2016.06.001

Shadmehr, R., and Mussa-Ivaldi, F. A. (1994). Adaptive representation of dynamics during learning of a motor task. J. Neurosci. 74, 3208-3224.

Shannon, C. E. (1949). Communication in the presence of noise. Proc. Inst. Radio Eng. 37, 10-21. doi: 10.1109/JRPROC.1949.232969

Song, M. H., and Godøy, R. I. (2016). How fast is your body motion? Determining a sufficient frame rate for an optical motion tracking system using passive markers. PLoS ONE 11:e150993. doi: 10.1371/journal.pone.0150993

Starkes, J., Helsen, W., and Elliott, D. (2002). A ménage à trois: the eye, the hand and on-line processing. J. Sports Sci. 20, 217-224. doi: 10.1080/026404102317284772

Terrier, R., Forestier, N., Berrigan, F., Germain-Robitaille, M., Lavallière, M., and Teasdale, N. (2011). Effect of terminal accuracy requirements on temporal gazehand coordination during fast discrete and reciprocal pointings. J. Neuroeng. Rehabil. 8, 10-30. doi: 10.1186/1743-0003-8-10

Vernazza, S., Alexandrov, A., and Massion, J. (1996). Is the center of gravity controlled during upper trunk movements? Neurosci. Lett. 206, 77-80 doi: 10.1016/S0304-3940(96)12464-2

Vernazza-Martin, S., Martin, N., and Massion, J. (2000). Kinematic synergy adaptation to microgravity during forward trunk movement. J. Neurophysiol. $83,453-464$.

Watt, D. G. D. (1997). Pointing at memorized targets during prolonged microgravity. Aviat. Space Environ. Med. 68, 99-103.

White, O., Lefèvre, P., Wing, A. M., Bracewell, R. M., and Thonnard, J.-L. (2012). Active collisions in altered gravity reveal eye-hand coordination strategies. PLoS ONE 7:e44291. doi: 10.1371/journal.pone.0044291

Woodworth, R. S. (1899). The accuracy of voluntary movements. Psychol. Rev. Monogr. Suppl. 3, 1-114.

Wolpert, D. M., and Ghahramani, Z. (2000). Computational principles of movement neuroscience. Nat. Neurosci. 3, 1212-1217. doi: 10.1038/81497

Wolpert, D. M., and Kawato, M. (1998). Multiple paired forward and inverse models for motor control. Neural Netw. 11, 1317-1329. doi: 10.1016/S0893-6080(98)00066-5

Conflict of Interest Statement: The authors declare that the research was conducted in the absence of any commercial or financial relationships that could be construed as a potential conflict of interest.

Copyright (C) 2017 Macaluso, Bourdin, Buloup, Mille, Sainton, Sarlegna, Vercher and Bringoux. This is an open-access article distributed under the terms of the Creative Commons Attribution License (CC BY). The use, distribution or reproduction in other forums is permitted, provided the original author(s) or licensor are credited and that the original publication in this journal is cited, in accordance with accepted academic practice. No use, distribution or reproduction is permitted which does not comply with these terms. 